\title{
Transformation From Asymptomatic to Symptomatic of Lower Cervical Spinal Dural Arteriovenous Fistula
}

\author{
-Case Report-
}

\author{
Toru SASAMORI, ${ }^{1}$ Kazutoshi HIDA, ${ }^{1}$ Takeshi ASANO, ${ }^{1}$ \\ Toshiya OSANAI, ${ }^{1}$ Syunsuke YANO, ${ }^{2}$ Takeshi AOYAMA, ${ }^{1}$ \\ Yoshinobu IWASAKI, ${ }^{2}$ and Kiyohiro HOUKIN ${ }^{1}$ \\ ${ }^{1}$ Department of Neurosurgery, Hokkaido University Graduate School of Medicine, \\ Sapporo, Hokkaido; \\ ${ }^{2}$ Department of Neurosurgery, Sapporo Azabu Neurosurgical Hospital, Sapporo, Hokkaido
}

\begin{abstract}
A 60-year-old woman presented with a spinal dural arteriovenous fistula (SDAVF) located in the lower cervical region, which had been asymptomatic for 56 months after the initial detection. She underwent embolization of the SDAVF when she became symptomatic, but her neurological recovery was only partial. Cervical SDAVF is rare but sometimes discovered in an asymptomatic state. The natural history and validity of preventive treatment for asymptomatic SDAVF have not been fully established. Her unfavorable outcome demonstrates the potential advantages of adequate treatment at an early stage even for asymptomatic SDAVF.
\end{abstract}

Key words: asymptomatic fistula, cervical spine, natural history, spinal dural arteriovenous fistula

\section{Introduction}

Spinal dural arteriovenous fistula (SDAVF) is a rare disorder in which an abnormal arteriovenous shunt develops between one or more dural branches of the radicular artery and a single radiculomedullary vein, typically within the dorsal surface of the dural root sleeve in the intervertebral foramen. ${ }^{1)}$ Most SDAVFs are found in the thoracolumbar region, whereas cervical and sacral lesions occur in only $2 \%$ and $4 \%$ of patients, respectively. ${ }^{5)}$ Congestive myelopathy due to chronic venous hypertension is the most common pathological condition contributing to neurological deficits in patients with SDAVF.2) Subarachnoid hemorrhage (SAH) occurs in approximately $45 \%$ of patients with cervical SDAVF, and only rarely in thoracolumbar regions. ${ }^{5}$ Asymptomatic SDAVF is common in the cervical region, but is rare in the thoracolumbar region. ${ }^{5,7)}$ The natural history and validity of preventive treatment for asymptomatic SDAVF have not been fully discussed. We report a case of a 60-year-old woman with a lower cervical SDAVF which was asymptomatic at first, but became symptomatic and was embolized approximately 5 years later.

\section{Case Report}

A 60-year-old woman was referred to our hospital on sus-

Received February 22, 2012; Accepted April 5, 2012 picion of spinal arteriovenous malformation at the craniovertebral junction, based on abnormal findings on brain magnetic resonance (MR) imaging performed for head trauma. On admission, neurological examination revealed no definite abnormality. $\mathrm{T}_{2}$-weighted $\mathrm{MR}$ imaging revealed a marked flow void on the dorsal surface of the spinal cord from the craniovertebral junction to $\mathrm{C} 4$ (Fig. 1A). No intramedullary abnormality was detected. Spinal angiography revealed an SDAVF in which an abnormal arteriovenous connection had developed between multiple dural branches of the C5 radicular artery from the left ascending cervical artery, and a single radiculomedullary vein at the C4-5 intervertebral level (Fig. 1B, C). We proposed embolization of the fistula to avoid predictable risks such as congestive myelopathy and $\mathrm{SAH}$, which could cause irreversible neurological deficits. However, she refused the proposed treatment because she had no related symptoms. Moreover, she did not return to our hospital after our diagnosis and proposed treatment.

Sixty-two months later, she visited our hospital again with complaints of a 6-month history of progressive gait disturbance, difficulty in elevating her arms, and sensory disturbance in her lower extremities. Neurological examination revealed weakness of the upper extremities (manual muscle test 4/5), spastic paraparesis (3/5) including ankle clonus and pathological reflexes, and decreased sensation to pin prick and light touch below the T2 dermatome. She was barely able to walk by herself with a cane. Bladder dysfunction was also detected. $\mathrm{T}_{2}$-weighted $\mathrm{MR}$ 
imaging showed not only remarkable flow void along the cervical spinal cord, but also marked swelling of the spinal cord with intramedullary high intensity from the C2 to T3 levels (Fig. 2A). Spinal angiography revealed an SDAVF supplied by multiple tiny feeders from the left ascending cervical artery at the C4-5 level, which appeared to be

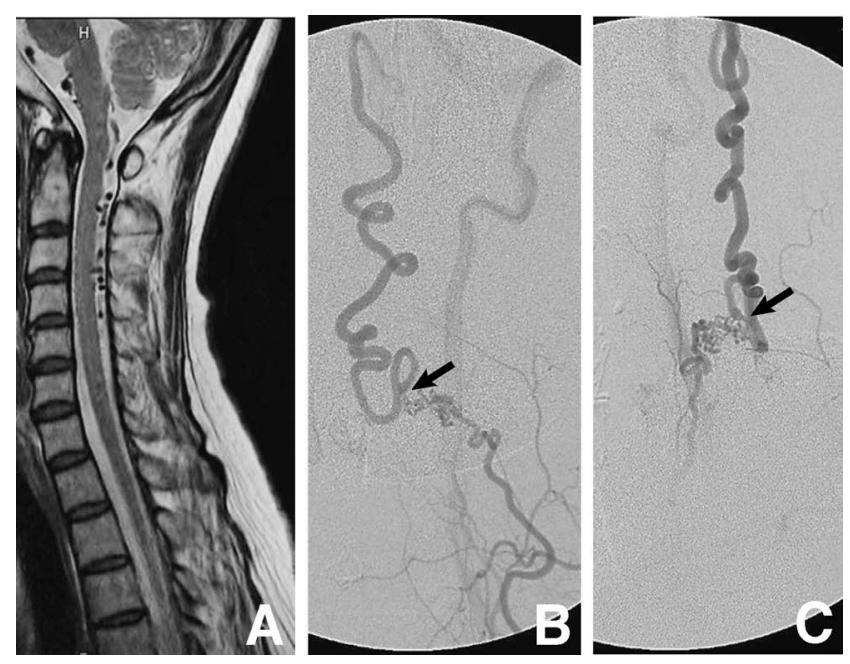

Fig. 1 Neuroimaging findings obtained during the asymptomatic period. A: $T_{2}$-weighted magnetic resonance image revealing a remarkable flow void on the dorsal surface of the cervical spinal cord, without intramedullary change. B, C: Anteroposterior (B) and lateral (C) selective angiograms of the left ascending cervical artery showing the spinal dural arteriovenous fistula supplied by multiple tiny feeders from the left $\mathrm{C} 5$ radicular artery at the C4-5 level (arrows), and draining into the dilated perimedullary vein along the dorsal cervical cord. similar to the past findings, except for new caudal drainage along the dorsal surface of the spinal cord (Fig. 2B-D). Computed tomography with contrast medium showed an SDAVF located within the dorsal surface of the dural root sleeve in the left C4-5 intervertebral foramen (Fig. 3A).
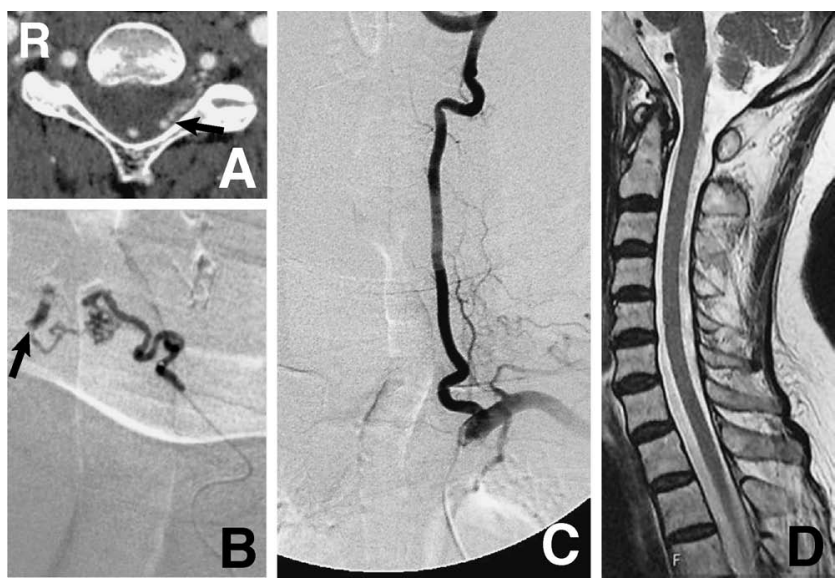

Fig. 3 A: Computed tomography scan with contrast medium demonstrating a spinal dural arteriovenous fistula located within the dorsal surface of the dural root sleeve in the left C4-5 intervertebral foramen (arrow). B: Superselective angiogram of the left $\mathrm{C} 5$ radicular artery demonstrating the glue cast from the feeders via the fistula into the proximal draining vein. The fistula point was indicated by the marked caliber change (arrow). C: Postoperative angiogram of the left subclavian artery performed 6 months after embolization showing complete obliteration of the fistula. $\quad \mathrm{D}: \mathrm{T}_{2}$-weighted magnetic resonance image performed 44 months after embolization showing no findings indicative of recurrence.
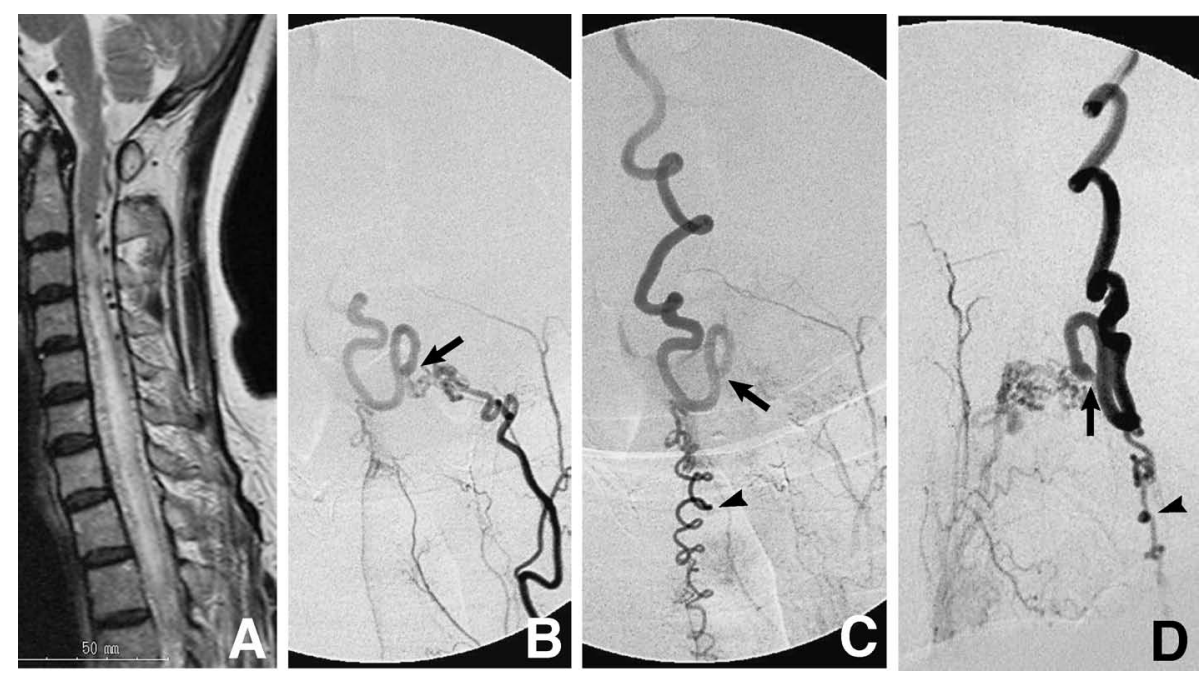

Fig. 2 Neuroimaging findings obtained just before embolization. A: $\mathbf{T}_{2}$-weighted magnetic resonance image showing marked swelling of the spinal cord with intramedullary high intensity from C2 to T3 levels and multiple flow voids along the dorsal surface of the cervical cord. B-D: Anteroposterior (B, C) and lateral (D) selective angiograms of the left ascending cervical artery showing the spinal dural arteriovenous fistula supplied by multiple tiny feeders from the left C5 radicular artery at the C4-5 level (arrows). Note the dorsal perimedullary vein flow in the caudal direction, which was not detected before (arrowheads). 
At approximately 6 months after onset, she underwent embolization using n-butyl 2-cyanoacrylate, and subsequent angiography revealed complete disappearance of the SDAVF (Fig. 3B). Her neurological condition gradually improved after embolization, and she was able to walk by herself without using a cane 3 months after embolization. Micturition disorder also improved, but partially persisted. Unfortunately, her neurological recovery was only partial even 6 months after embolization, although spinal angiography revealed no evidence of residual or recurrent SDAVF (Fig. 3C). Since then, she has exhibited no additional improvement of her neurological condition (Fig. 4). She was still suffering from gait disturbance, numbness of the lower extremities, and mild dysuria after a follow-up period of 44 months, although MR imaging showed no findings indicative of recurrence (Fig. 3D).

\section{Discussion}

Even today, the natural history of an asymptomatic SDAVF remains unclear, so the necessity for treatment is uncertain. Preventive therapy is the most beneficial option, since we cannot, with certainty, predict the onset of symptoms or the potential risks such as congestive myelopathy or SAH. Moreover, we know that neurological improvement after adequate treatment is often partial, once the patient becomes symptomatic. ${ }^{8)}$

Only 3 cases of asymptomatic SDAVF, including our

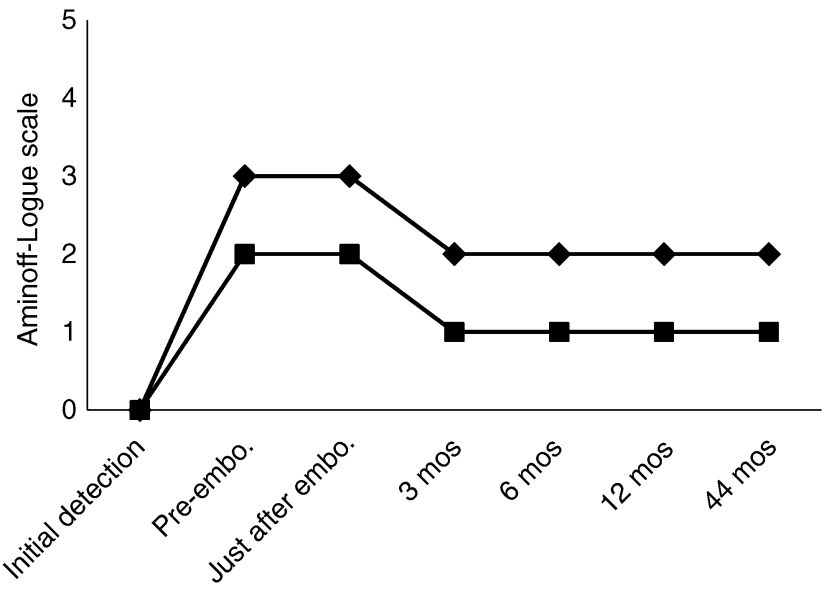

Fig. 4 Graph showing the time course of gait and micturition grade scored by the Aminoff-Logue scale. Filled diamonds and squares represent gait and micturition scores, respectively. case, have been reported (Table 1). ${ }^{3,6}$ A 56 -year-old man had an asymptomatic SDAVF at the T6 level. ${ }^{3)}$ Similar to our case, he underwent surgery when he became symptomatic after a 2-year asymptomatic period, but neurological improvement was only partial at 6 months after the treatment. These unfavorable clinical outcomes, including our case, demonstrate the importance of adequate treatment at the early stage even for an asymptomatic SDAVF. On the other hand, spontaneous disappearance of an SDAVF was detected after a 5-year asymptomatic period. ${ }^{6}$

Chronic venous hypertension is well known to be responsible for congestive myelopathy in patients with SDAVFs. ${ }^{2)}$ However, the exact etiology of the SDAVF remains unclear and there is also little information about the pathophysiology of asymptomatic SDAVF. ${ }^{3)}$ In the present case, her natural history provided us with valuable information. For example, the asymptomatic cervical SDAVF was present at least several years before becoming symptomatic. Only 3 cases, including the present case, described the asymptomatic period of SDAVF (Table 1). ${ }^{3,6)}$ However, the true asymptomatic period of SDAVF may be longer, because we still do not know when the morphological arteriovenous fistulas first developed. Today, we can make a diagnosis of asymptomatic SDAVF based on the observation of marked dilation of the perimedullary vein.

In our case, the chronological series of spinal angiograms before embolization revealed that an additional venous drainage route had newly developed when the patient became symptomatic (Fig. 1B, C). Whether this change was caused by prolonged venous hypertension, and/or by novel impairment of venous outflow remains unclear. However, the spinal angiogram in the venous phase revealed no additional changes to the venous outflow, including its occlusion, except for the caudal drainage along the spinal cord. Therefore, we think that this finding may be a compensatory change caused by prolonged venous hypertension. Dilated perimedullary vein in the asymptomatic period may reflect the presence of venous congestion. Even if the patient is asymptomatic, venous congestion may be present that gradually affects the spinal cord. Therefore, we would emphasize the importance of adequate treatment at an early stage even for an asymptomatic SDAVF.

Spinal cord changes in cases of SDAVF, including necrosis of the gray and white matter, have been attributed to venous congestion. ${ }^{4)}$ The onset of congestive myelopathy may reflect the appearance of these irreversible changes of the spinal cord, since neurological improvement is often partial even after adequate treatment. ${ }^{8)}$ Thus,

Table 1 Summary of the reported cases of asymptomatic spinal dural arteriovenous fistula

\begin{tabular}{|c|c|c|c|c|c|}
\hline Author (Year) & $\begin{array}{l}\text { Age (yrs), } \\
\text { Sex }\end{array}$ & Level & $\begin{array}{l}\text { Asymptomatic } \\
\text { period }\end{array}$ & Onset pattern & Outcome (follow-up period) \\
\hline Meder et al. $(1995)^{6)}$ & $56, \mathrm{M}$ & T6 & 5 yrs & remain asymptomatic & disappearance of the fistula \\
\hline Houdart et al. $(2001)^{3)}$ & $56, \mathrm{M}$ & T6 & 2 yrs & progressive myelopathy & $\begin{array}{l}\text { partial improvement after surgery } \\
\text { (6 mos })\end{array}$ \\
\hline Present case & $60, \mathrm{~F}$ & $\mathrm{C} 4-5$ & $56 \mathrm{mos}$ & progressive myelopathy & $\begin{array}{l}\text { partial improvement after } \\
\text { embolization ( } 44 \text { mos) }\end{array}$ \\
\hline
\end{tabular}


treatments for symptomatic SDAVF are likely to have limitations. Some irreversible changes of the spinal cord might be avoided by providing appropriate treatments within the asymptomatic period. Surgery for asymptomatic SDAVFs is already known to achieve good outcome. ${ }^{7)}$ Therefore, we should not miss the opportunity to successfully treat SDAVF to prevent the risk of future deficits.

\section{Conflicts of Interest Disclosure}

The authors have no personal financial or institutional interest in any of the drugs, materials, or devices in the article. All authors who are members of The Japan Neurosurgical Society (JNS) have registered online Self-reported COI Disclosure Statement Forms through the website for JNS members.

\section{References}

1) Aminoff MJ, Logue V: The prognosis of patients with spinal vascular malformations. Brain 97: 211-218, 1974

2) Hassler W, Thron A, Grote EH: Hemodynamics of spinal dural arteriovenous fistulas. An intraoperative study. J Neurosurg 70: 360-370, 1989

3) Houdart E, Redondo A, Saint-Maurice JP, Merland JJ: Natural history of an incidentally discovered spinal dural arteriovenous fistula. Neurology 57: 742-743, 2001

4) Hurst RW, Kenyon LC, Lavi E, Raps EC, Marcotte P: Spinal dural arteriovenous fistula: the pathology of venous hypertensive myelopathy. Neurology 45: 1309-1313, 1995

5) Jellema K, Tijssen CC, van Gijn J: Spinal dural arteriovenous fistulas: a congestive myelopathy that initially mimics a peripheral nerve disorder. Brain 129: 3150-3164, 2006

6) Meder JF, Devaux B, Merland JJ, Frédy D: Spontaneous disappearance of a spinal dural arteriovenous fistula. AJNR Am J Neuroradiol 16: 2058-2062, 1995

7) Shinoyama M, Endo T, Takahash T, Shimizu H, Takahashi A, Suzuki M, Tominaga T: Long-term outcome of cervical and thoracolumbar dural arteriovenous fistulas with emphasis on sensory disturbance and neuropathic pain. World Neurosurg 73: 401-408, 2010

8) Steinmetz MP, Chow MM, Krishnaney AA, Andrews-Hinders D, Benzel EC, Masaryk TJ, Mayberg MR, Rasmussen PA: Outcome after the treatment of spinal dural arteriovenous fistulae: a contemporary single-institution series and meta-analysis. Neurosurgery 55: 77-87, 2004

Address reprint requests to: Toru Sasamori, MD, Department of Neurosurgery, Hokkaido University Graduate School of Medicine, North-15 West-7, Kita-ku, Sapporo 060-8638, Japan. 